Journal of Social Sciences (COES\&RJ-JSS)

ISSN (E): 2305-9249 ISSN (P): 2305-9494

Publisher: Centre of Excellence for Scientific \& Research Journalism, COES\&RJ LLC

Online Publication Date: $1^{\text {st }}$ October 2017

Online Issue: Volume 6, Number 4, October 2017

DOI: 10.25255/jss.2017.6.4.816.826

\title{
The Theory of Development in Islamic Economy
}

\begin{abstract}
Hazem Alwadi
Department of Business Economic

Faculty of Business Administration

Tafila Technical University, Jordan

Abstract:

Islamic economy depends on the theory economic development hypotheses through finding its supports which are: Implement Allah's legislation that ensure the happiness, and economic welfare, and which is the cause for long benevolent and live, hood, rains, knowledge. The implementation of Allah's legislation means: Every Muslim must be workable and effective, every Muslim must request the knowledge too, implement the economic function for Islamic nation, and to evaluate every productive project has effective issues for the whole Muslims, save the secure sense and safety for every Muslims, Islamic necessitate the implementation of economic independency away of economic. Versatilities, and Islamic allow us getting personal possessiveness, physical inventiveness for encourage increasing productivity, creativeness, and enhancement qualitative productivity, necessitate the preservation of physical and conceptual ambience depend on the Islamic base " no damage no damage", and the systematic of ethical values. Necessitate the gathering reserves for investment, implement the regimental Islamic markets though values, Islamic ethical principles.
\end{abstract}

\section{Keywords:}

Islam, Economic, Development.

\section{Citation:}

Alwadi, Hazem (2017); The theory of development in Islamic Economy; Journal of Social Sciences (COES\&RJ-JSS), Vol.6,No.4,pp:816-826; DOI: 10.25255/jss.2017.6.4.816.826. 


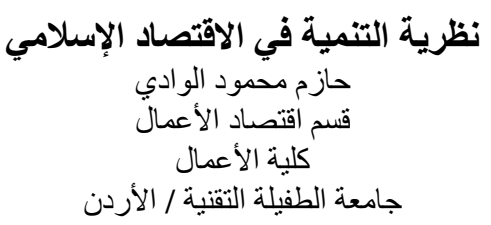

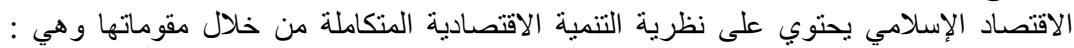

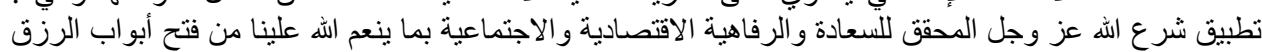

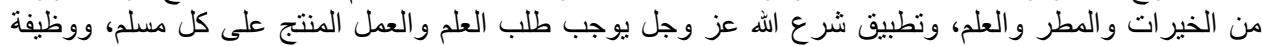

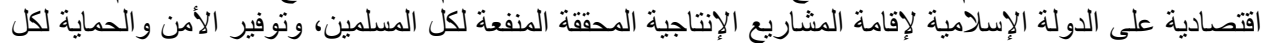

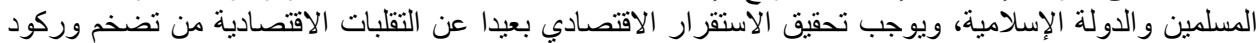

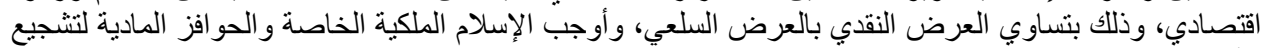

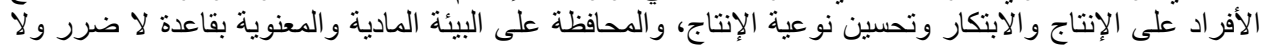

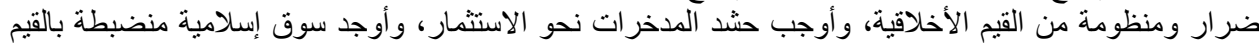
و الأخلاق الإسلامية .

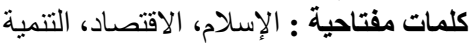

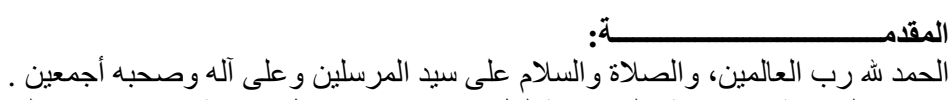

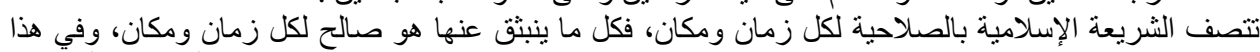

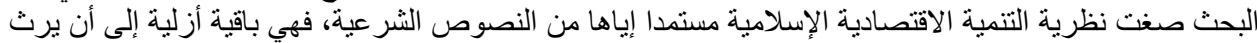

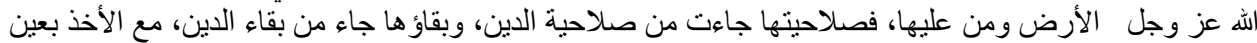

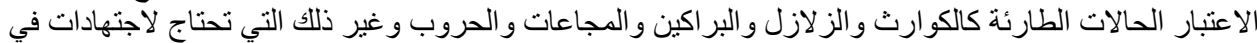

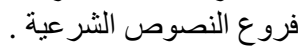

ونظرية التنمية الاقتصادية الإسلامية تقسم إلى جانبين هما :

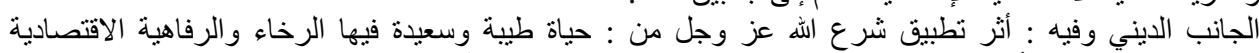

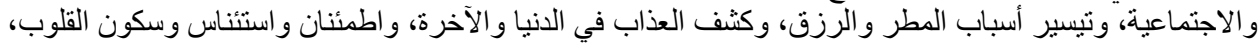

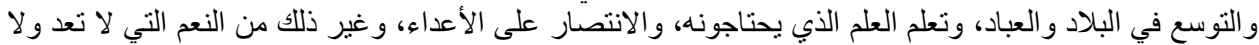

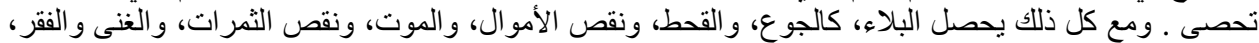
و غير ذللك.

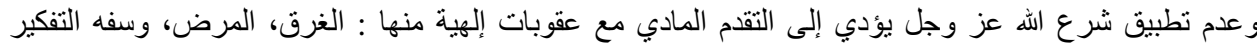

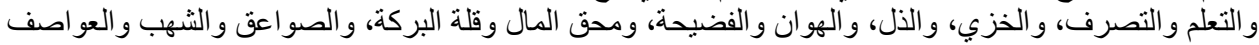

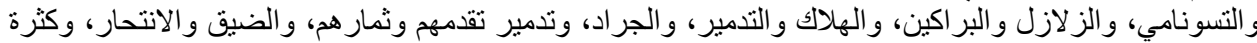
الأمر اض، و الحروب، والزلان، وغير ذلك .

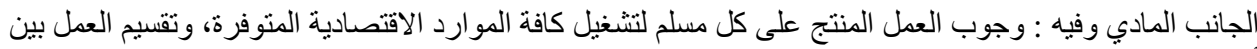

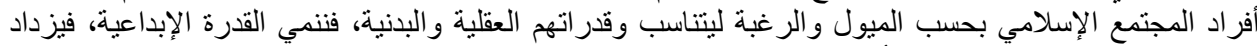

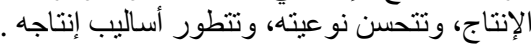

و وأوجب الإسلام الوظيفة الاقتصادية للدولة الإسلامية المتمثلة : بوضع الخطط اللازمة التإمة لتشغيل الموارد الاقتصـادية،

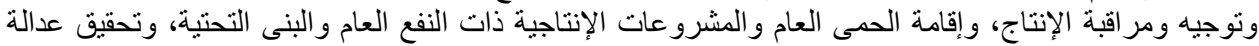

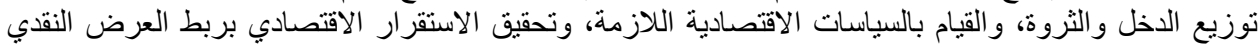

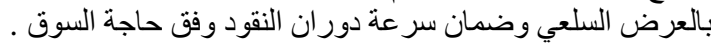

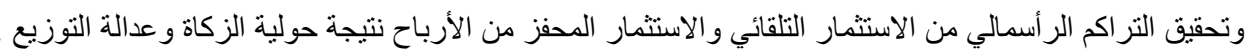

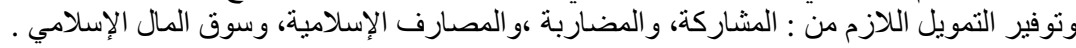


The theory of development in Islamic Economy

وتقديم الحو افز الدينية و المادية المحفزة للإنتاج وتطوره، بتثجيع الابتكار ات، والاختر اعات، ودعم التنظيم والإدارة الفنية .

وتوفير الأمن الداخلي والخارجي والاستقرار السياسي، و الحمى العام و إنشاء جيش قوي عددا و عدة، و إنشاء جهاز

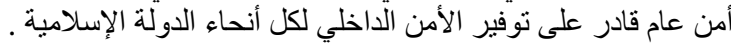
و المحافظة و عدم التعدي على القيم والأخلاق و العادات و التقاليد المتو افقة مع التعاليم الإسلامية .

وبذلك نكون ابتعدنا عن كل أسباب وسمات ومرتكز ات التخلف الاقتصادي، ووصلنا للتنمية الاقتصادية الإسلامية . أهمية البحث : تكمن أهمية البحث في إيجاد إطار نظري لنظرية التتمية الاقتصادية في الاقتصاد الإسلامي قابلة

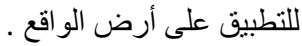

مشكلة البحث : كثرة الكتابات عن التتمية الاقتصادية من منظور إسلامي بشكل مفصل ومطول، و هذا يجعلنا بحاجة

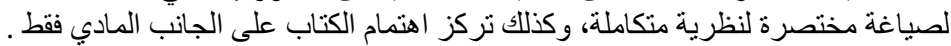

منهج البحث : اتبعت المنهج الاستقرائي، فقت بدر اسة النصوص الثرعية من القرآن الكريم والسنة النبوية الثريفة،

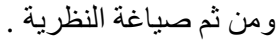

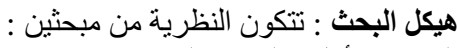

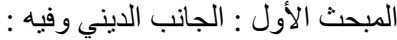

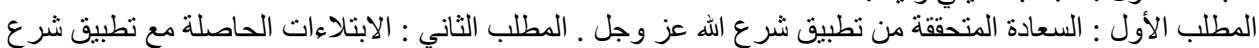

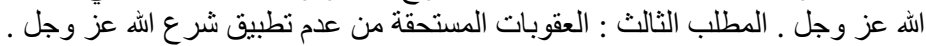

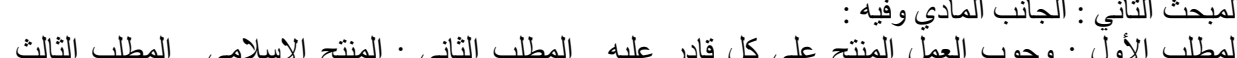

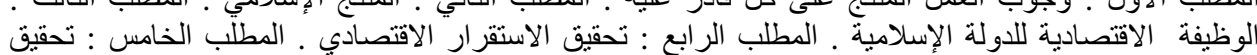

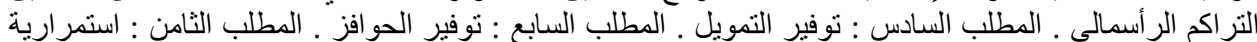

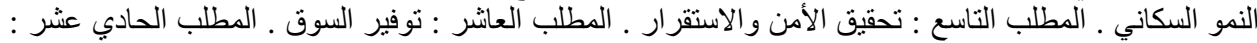

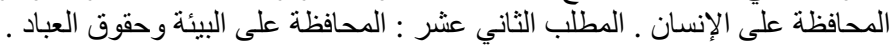

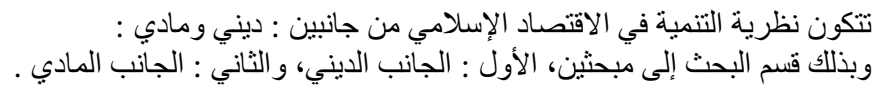

المبحث الأول : الجاتب الايني : يتكون من المطالب التالية :

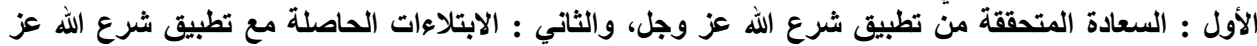

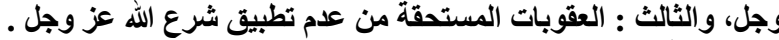
المطلب الأول : السعادة المتحققة من تطبيق شر ع عُ الله عز وجل :

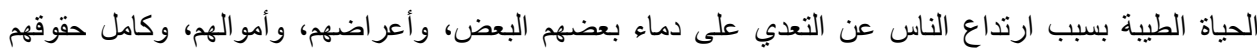

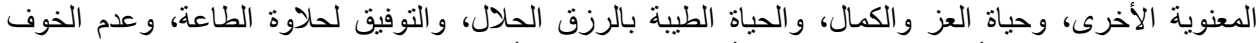

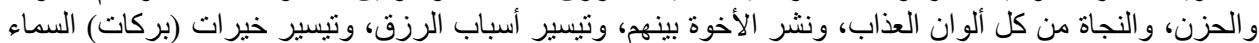

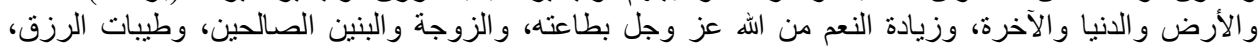

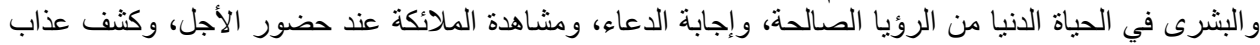

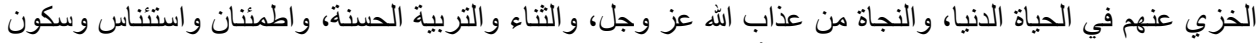

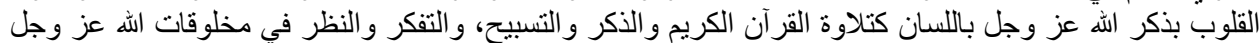

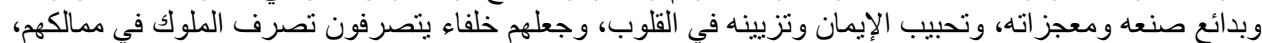

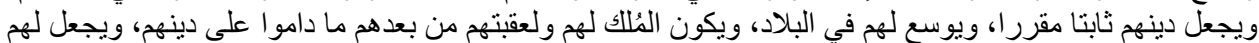

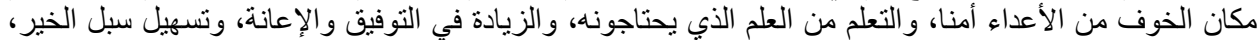

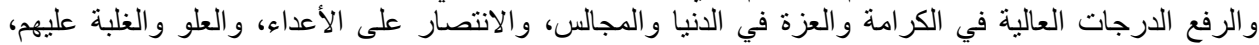




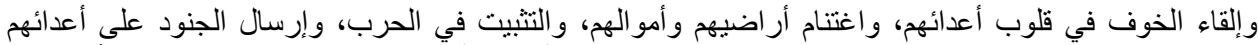

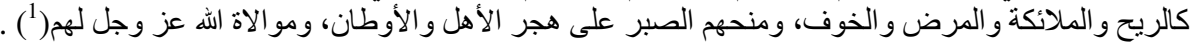

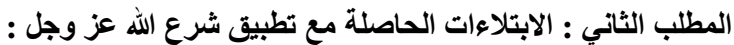

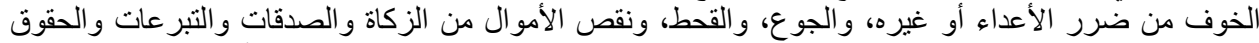

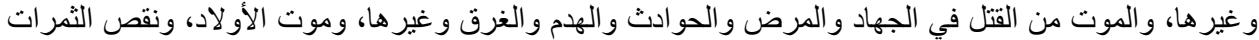

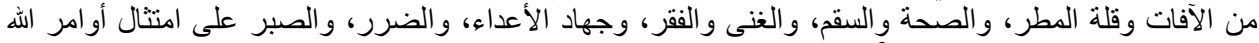

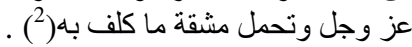

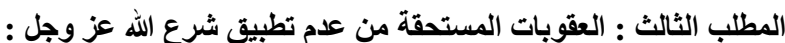

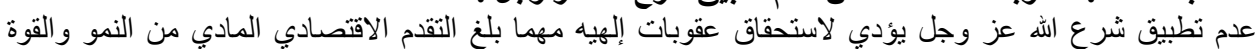

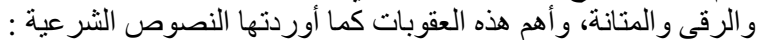

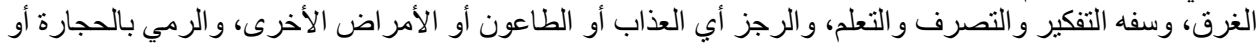

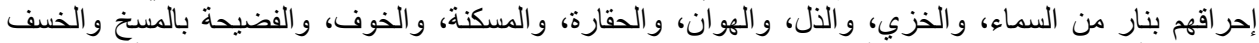

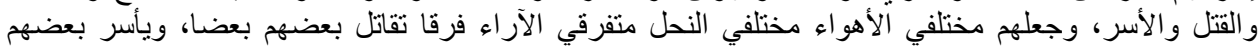

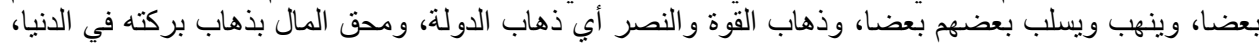

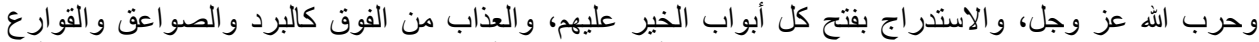

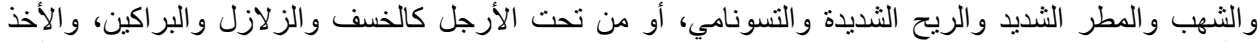

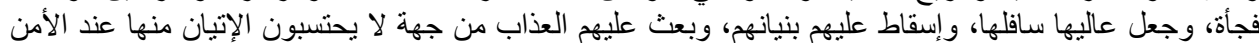

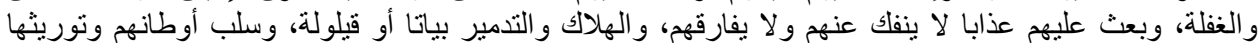

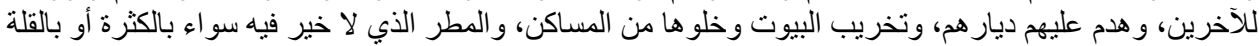

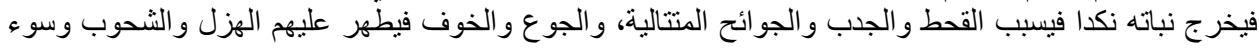

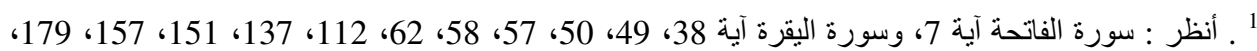

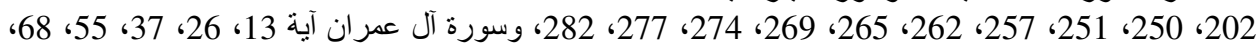

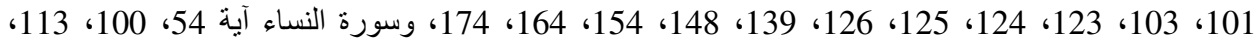

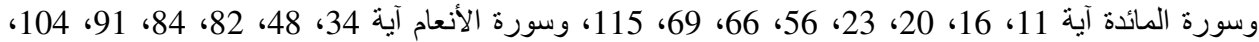

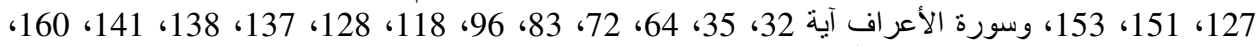

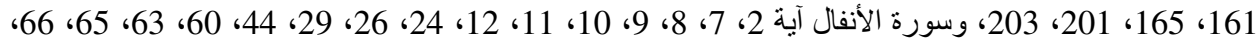

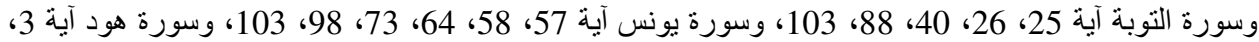

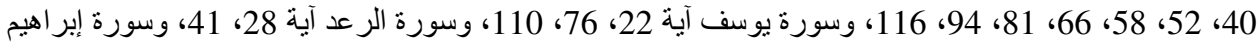

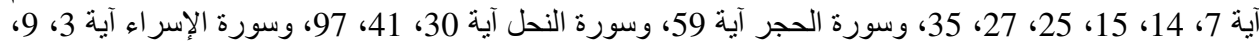

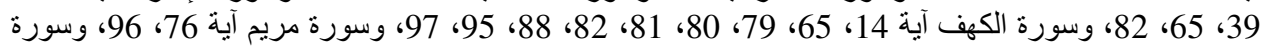

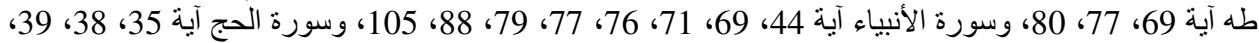

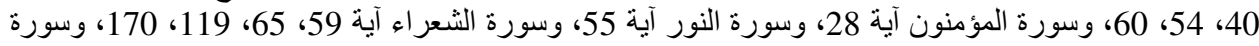

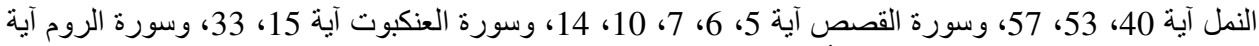

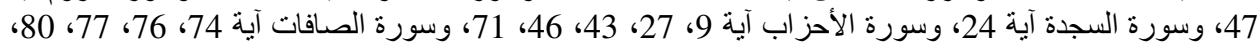

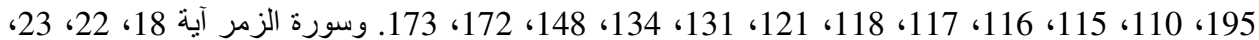

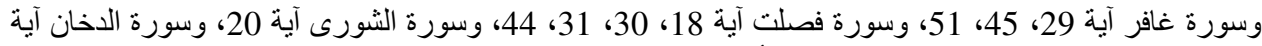

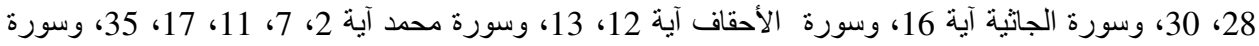

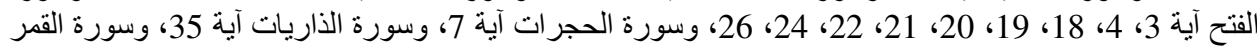

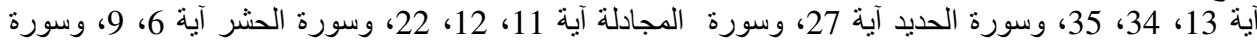

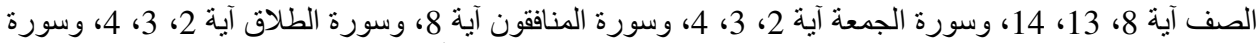

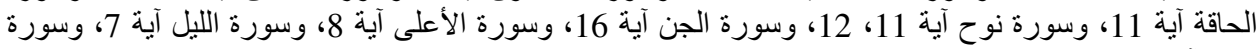
البينة آية 3 .

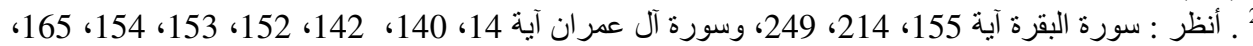

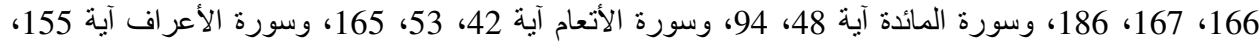

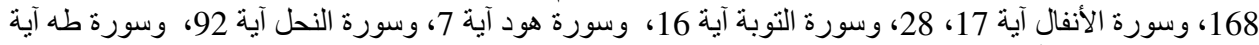

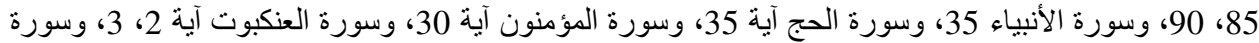

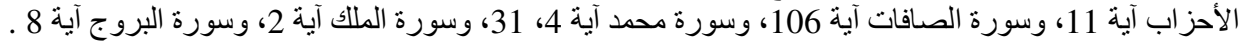


الحال و الفقر، و أمراض الدنيا ومصائبها و أوجاعها و أسقامها وبلاياها وذهاب الأموال والأو لادي، وفساد الأعمال

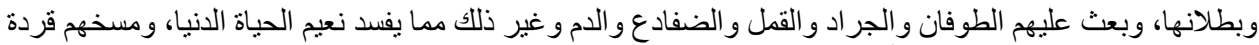

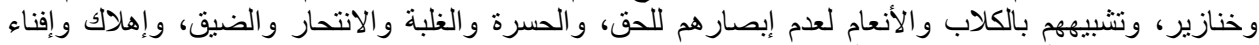

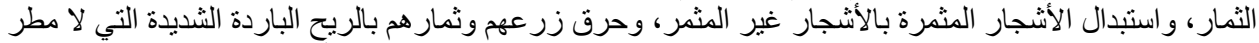

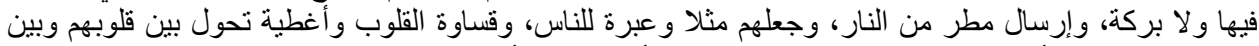

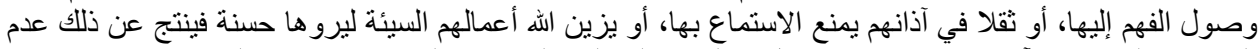

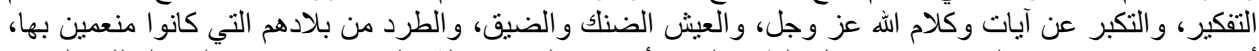

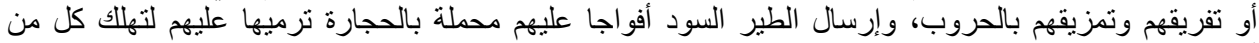

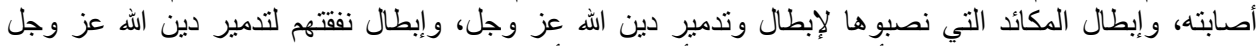

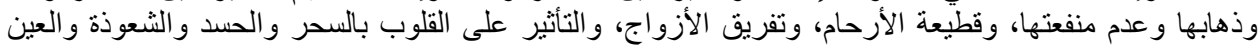

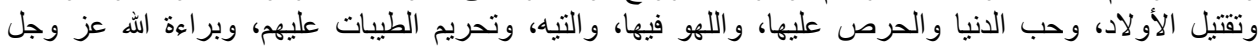

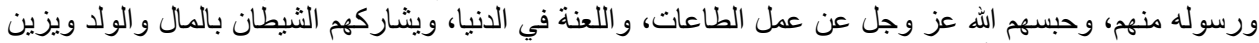

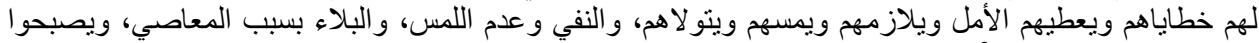

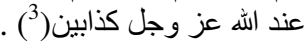

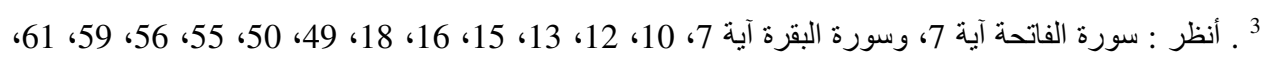

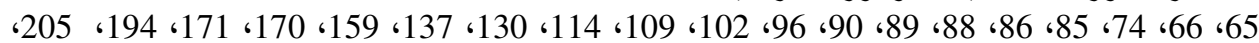

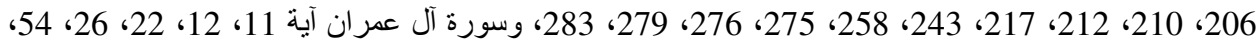

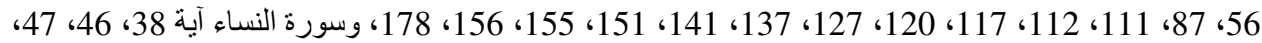

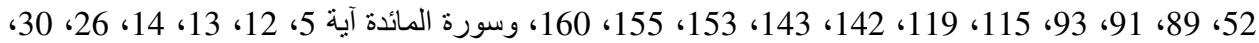

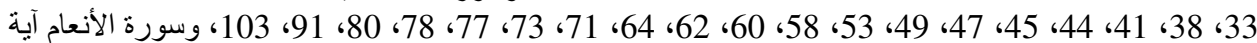

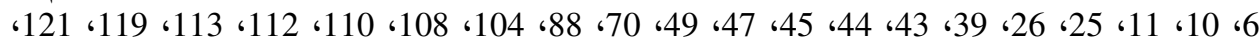

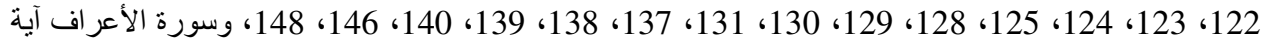

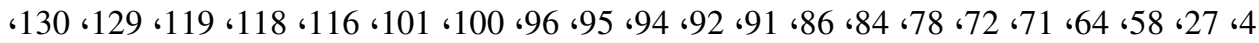
133، 134، 134، 136، 183

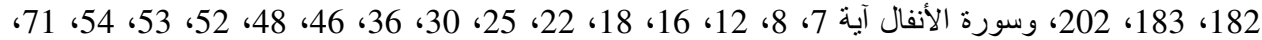

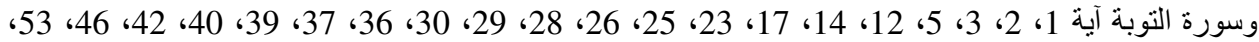

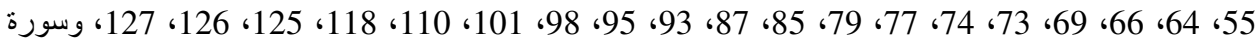

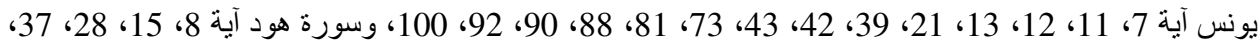

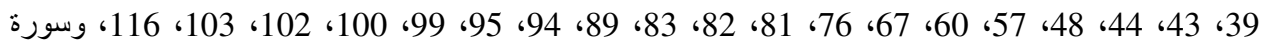

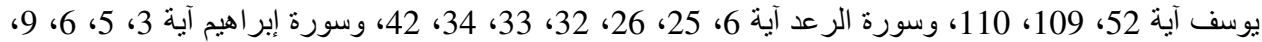

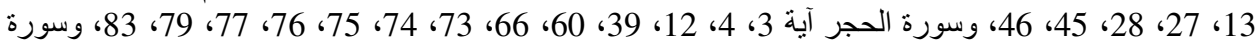

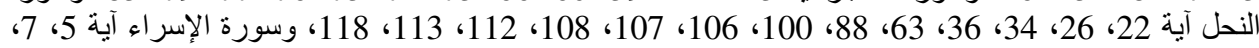

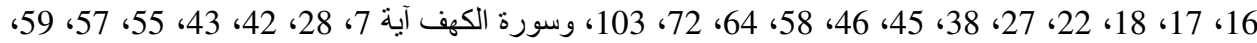

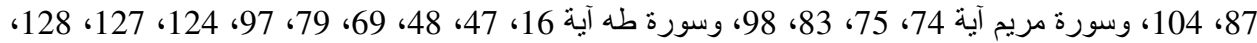

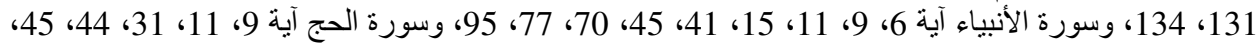

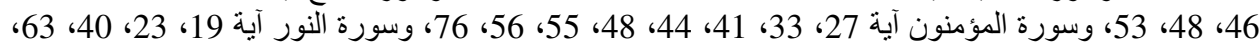

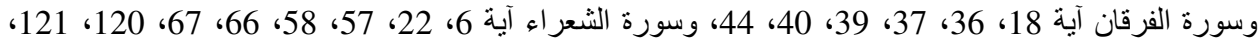

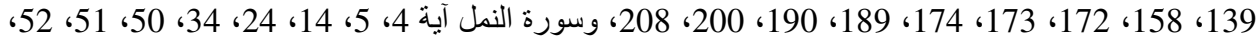

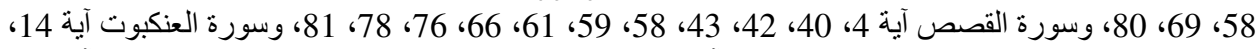

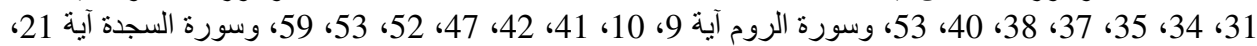

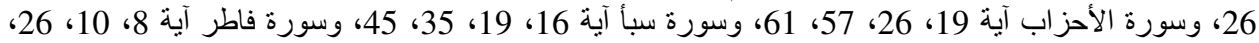

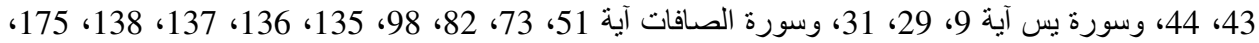

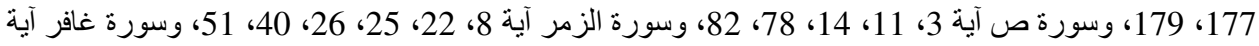

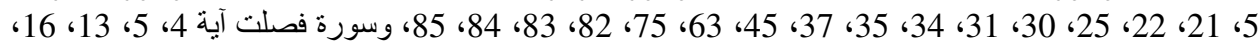

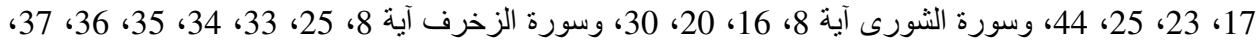

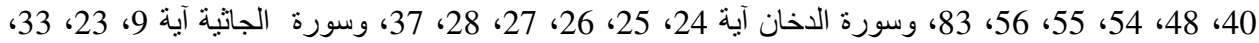

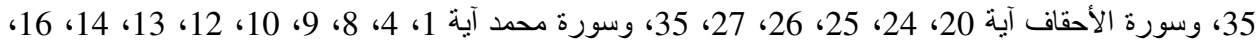

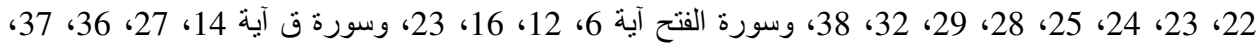

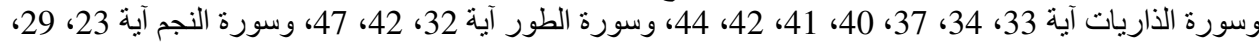




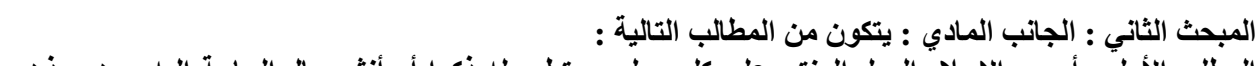

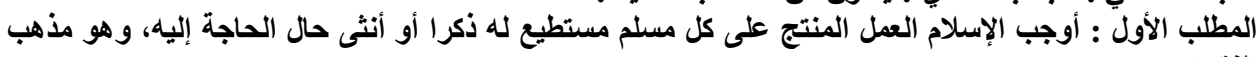

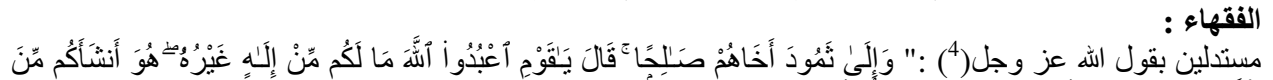

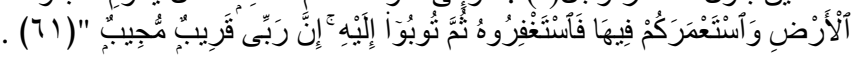

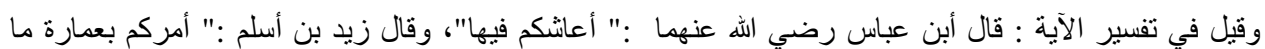

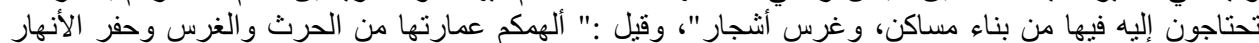

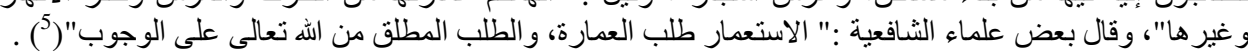

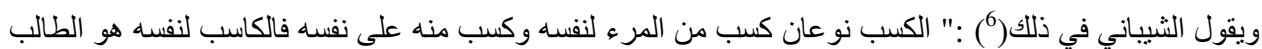

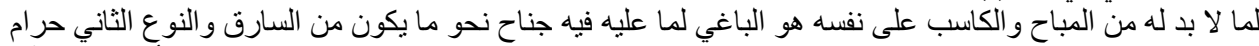

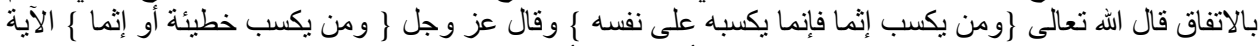

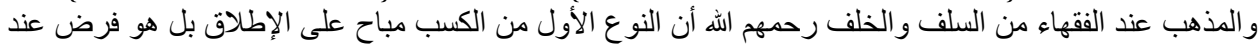

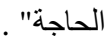

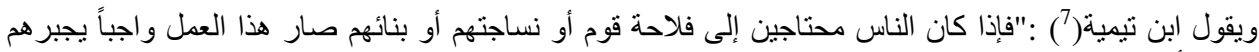

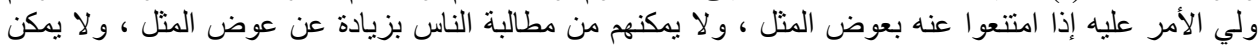

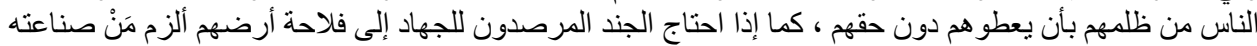

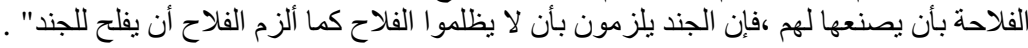

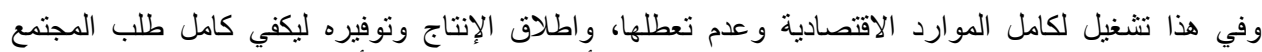

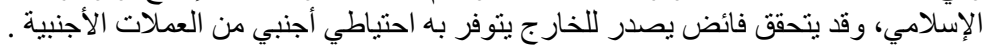

و الإسلام لم يوجب العمل على كل مسلم فقط، و إنما راعى الميول و الرغبة و القدرات العقلية والجسدية في اختبار

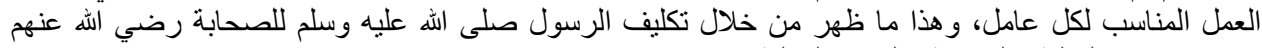

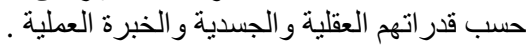

وفي هذا تقسيم للعمل بين أفراد المجتمع ليشمل كل القطاعات الاقتصادية المطلوبة، مما يجعل العامل ينتج أكبر كمية

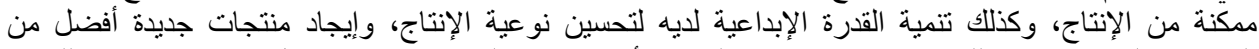

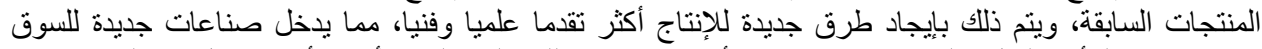

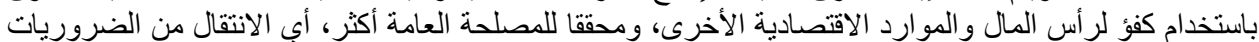

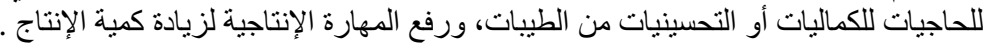

وفي هذا استخدام أمثل للموارد الاقتصادية، فيجعلها أكثر استغلالا واستخداما، وبعدا عن الهدر والإسراف وسوء الاستخدام

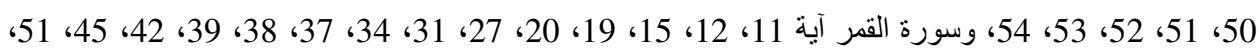

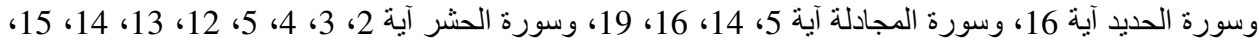

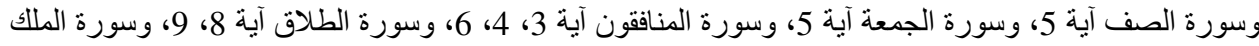

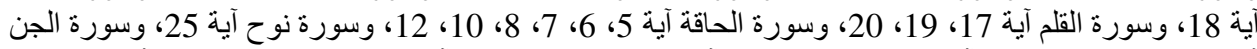

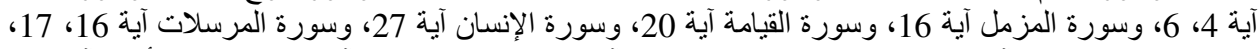

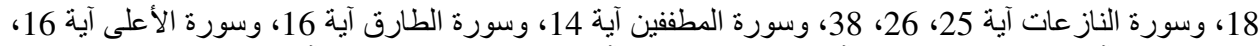

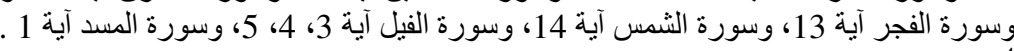

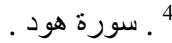

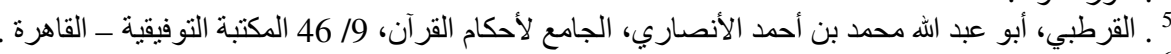

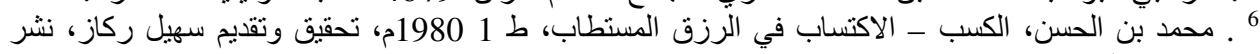

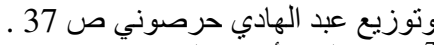
7 تقيي الدين أحمد، الحسبة في الإسلام، تحقيق تحقيق سيد بن محمد بن أبي سعدة، ط1 نشر وتوزيع مكتبة دار الأرقم ـ 
و هذا يتحقق أيضا من خلال : تحريم الربا الذب فيه متاجرة المال بالمال أو متاجرة المال بالزمن، وفي كلا الحالين

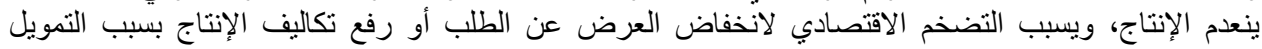

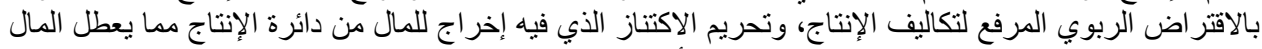

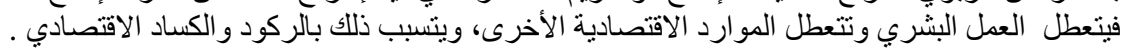

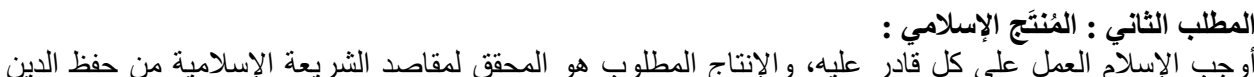

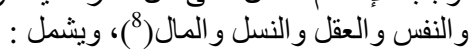

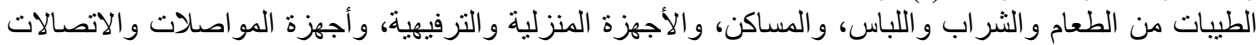

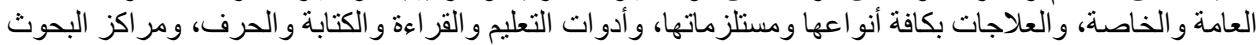

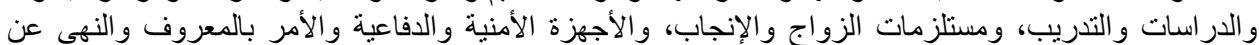
المنكر ونشر الدعوة الإسلامية، و إقامة البنى التحتية، وغير ها من حوائج الحياة الأساسية .

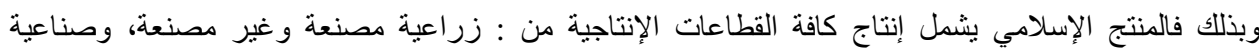

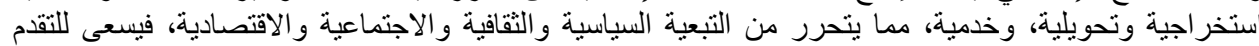
و التطور في الإنتاج و أسالييه ووسائله وطرقه و آلياته، و السعي للمشاريع ذات الإنتاجية كبيرة الحجم .

المطلب الثالث : الوظيفة الاقتصادية للاولة الإسلامية :

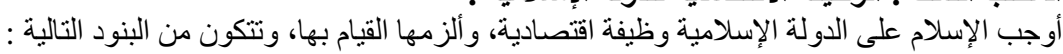
1.

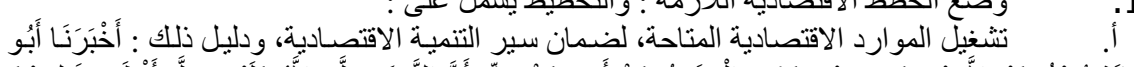

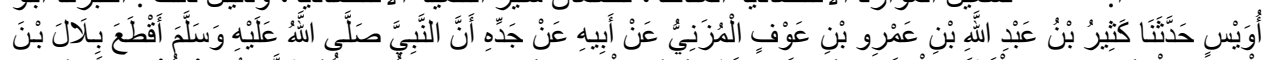

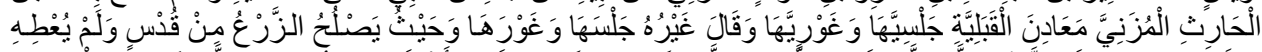

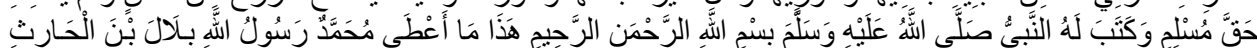

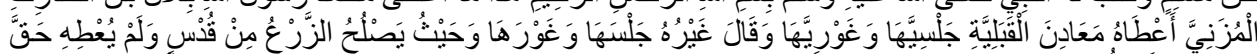

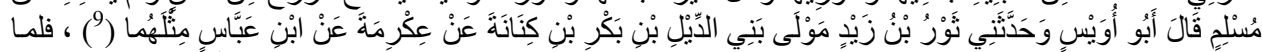

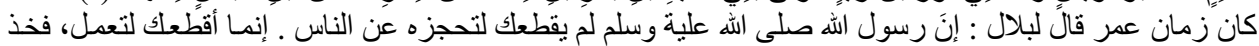

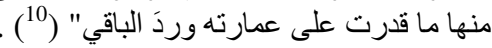

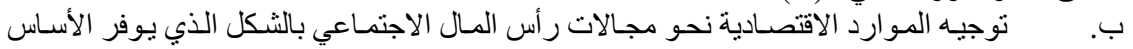

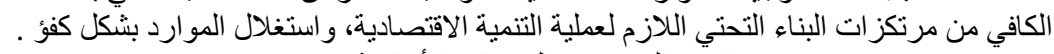

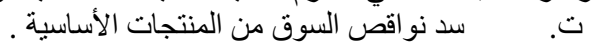

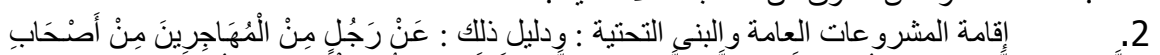

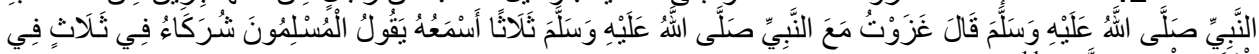

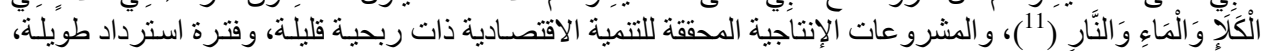

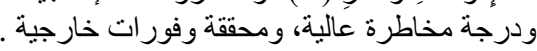

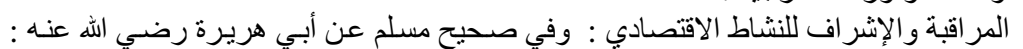

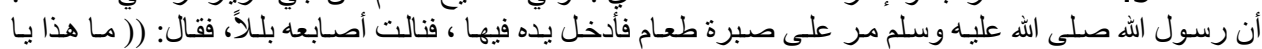

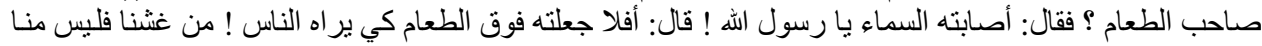

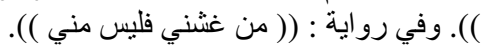

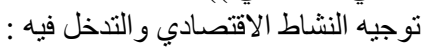
. 4 البدء بإنتاج الطيبات من الضروريات ثم الحاجيات ثم التحسينيان ومنع الخبائث من السوق الإسلامي .

8 8 . الثاطبي، أبو إسحق إبراهيم بن موسى، المو افقات في أصول الثريعة، حقق وعلق عليه : خالد عبد الفتاح شبل،

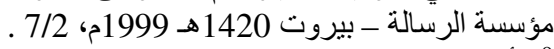

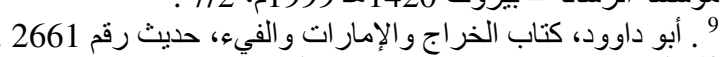
10 1 . أبو عبيد، القاسم بن سلام، كتاب الأمو ال، تحقيق وتعليق محمد خليل هراس، دار الكتب العلمية - بيروت، ط1

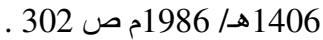




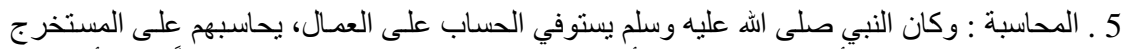

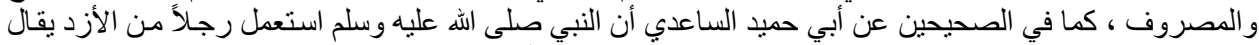

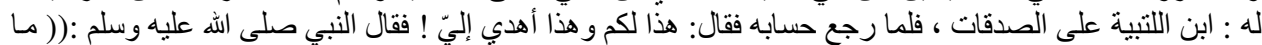

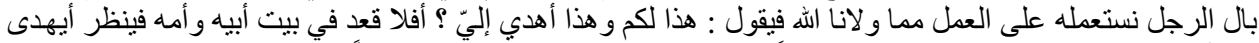

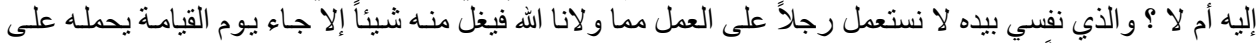

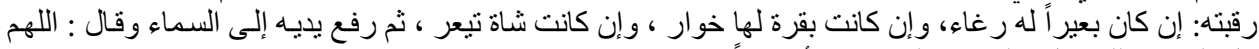

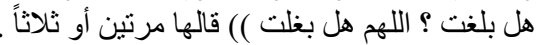

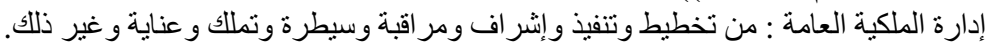

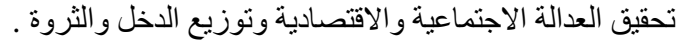

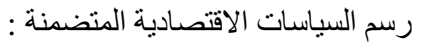

\section{.5} .6

أ. السياسة المالية : تهدف تحقيق الكفاءة بإنفاق الموارد المالية في مجالات التتمية الاقتصادية،

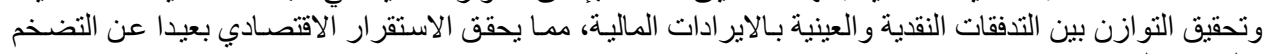

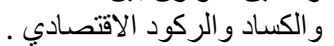

ب. السياسـة النقدية : تهدف لتوجيـه المدخر ات لاستثمار ات منتجة، و لإيجاد التوازن بين الطلب

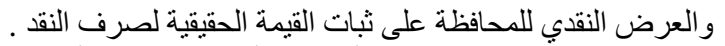

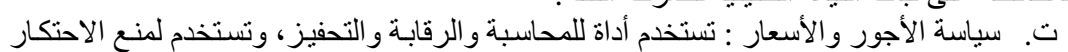

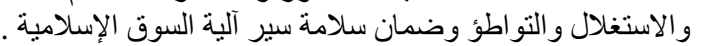

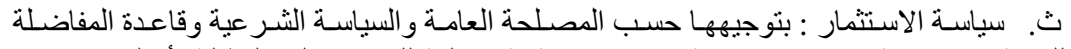

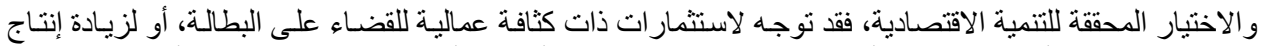

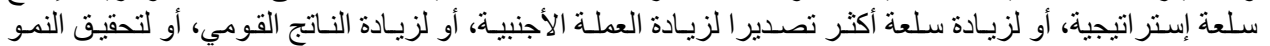

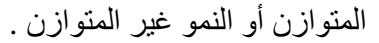

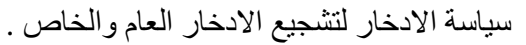

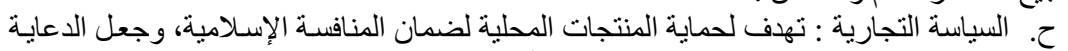

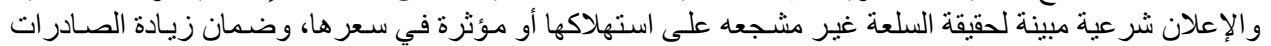

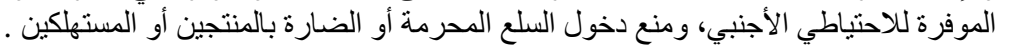
8.

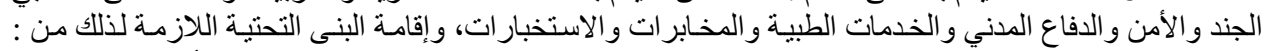

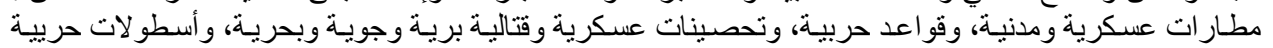

$$
\text { المطلب الرابع : الاستقرار الاقتصادي : }
$$
و غير ذلك .

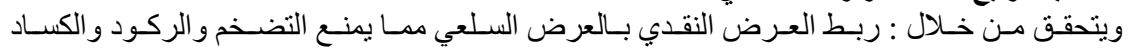

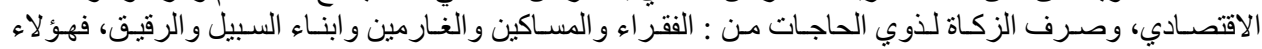

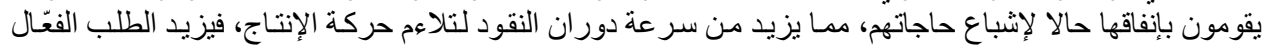

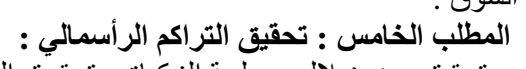
ويتسع مدى السوق .

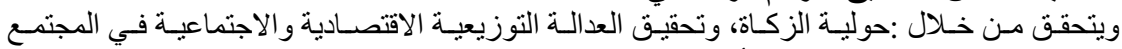

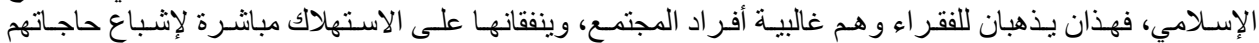

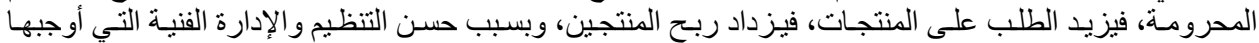

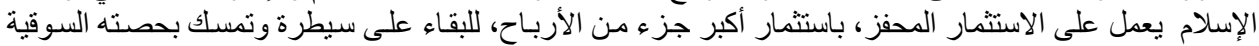

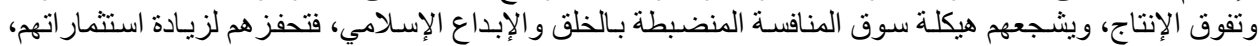

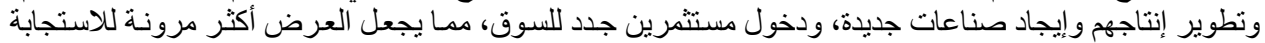

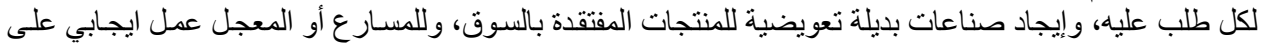

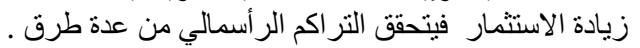

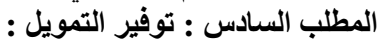

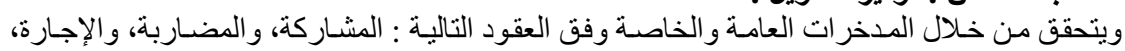

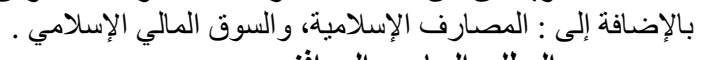
المطلب السابع : الحوافز : الإنة

تنقسم الحو افز في الاقتصاد الإسلامي إلى : الإلى : حو افز مادية، وحو افز دينية .

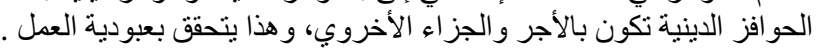




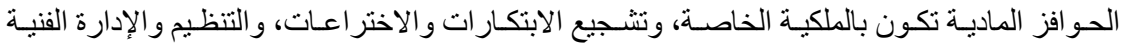

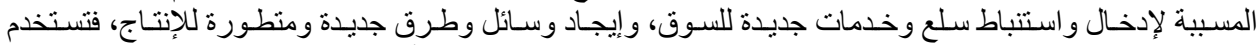

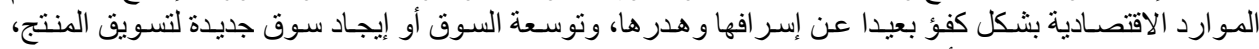

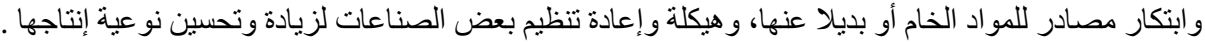

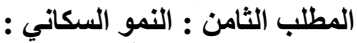

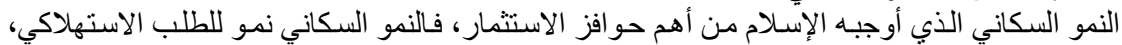

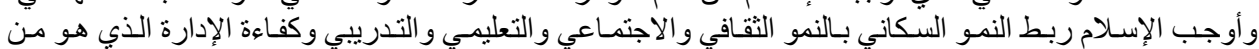

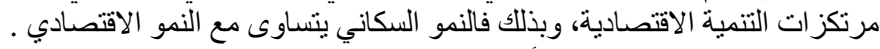

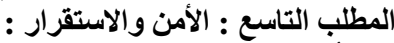

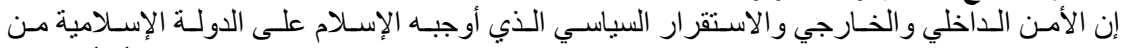

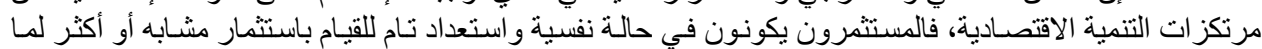

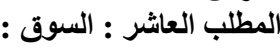

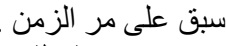

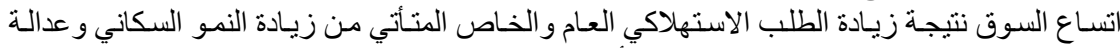

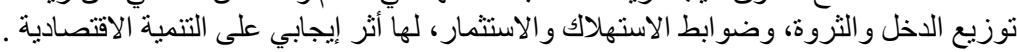

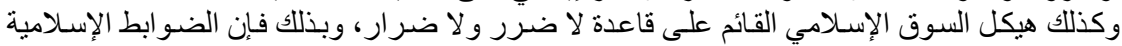

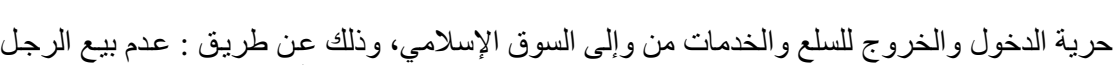

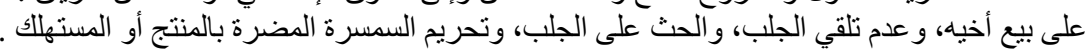

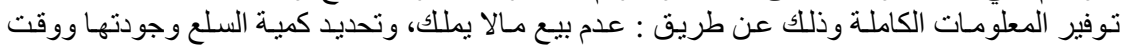

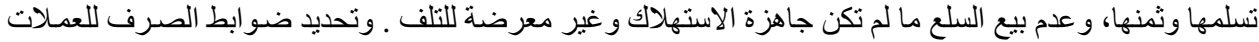

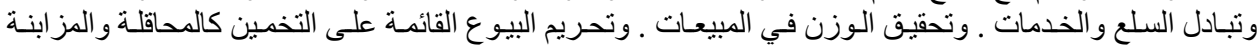

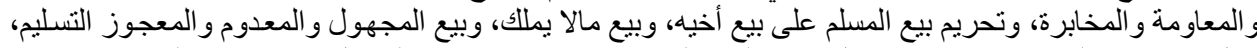

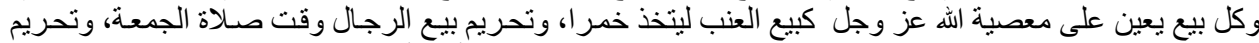

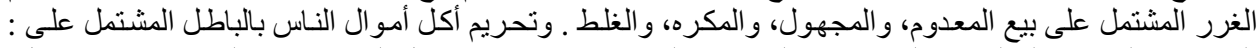

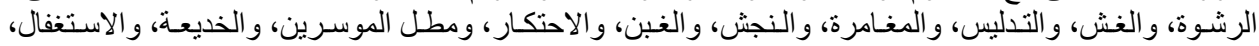

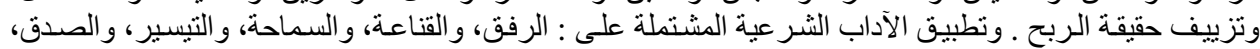

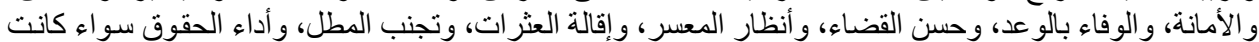

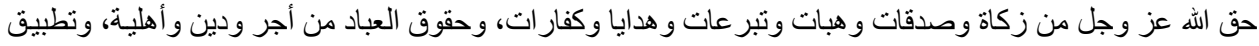

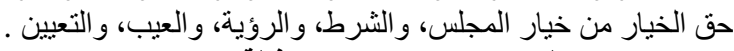

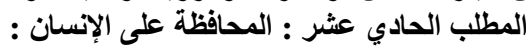

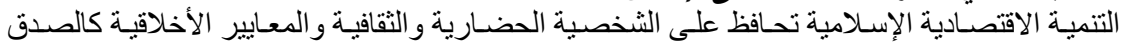

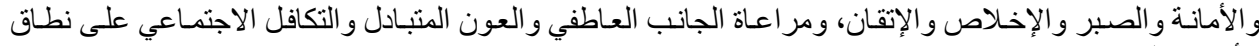

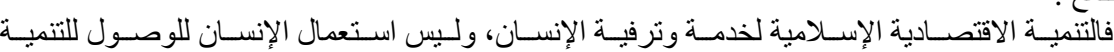
الأسرة و المجتمع .

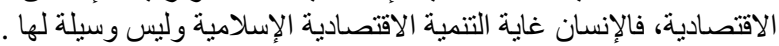

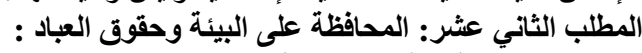

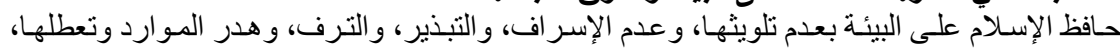

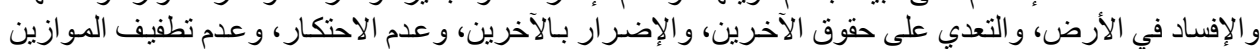

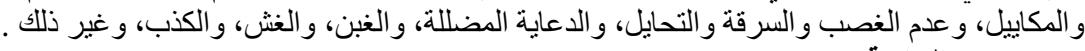

الخاتمة الغنان

تتكون الخاتمة من النتائج والتوصيات . النتائج :

نظرية التنمية الاقتصادية الإسلامية تتكون من جانبين : ديني ومادي . 2.

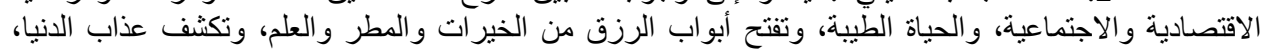

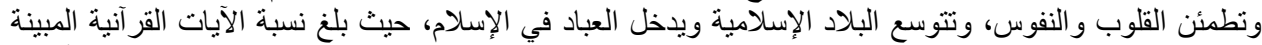

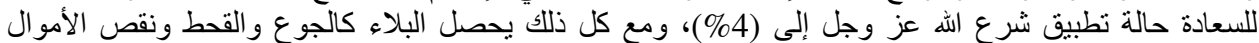

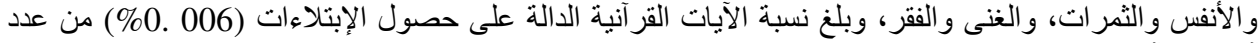
آيات القرآن الكريم . 


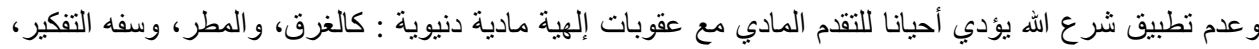

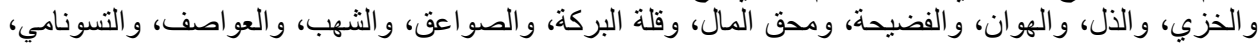

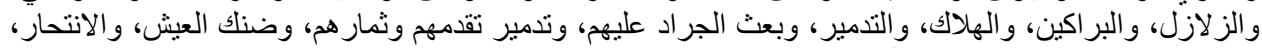

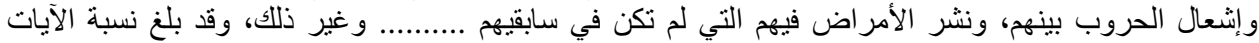

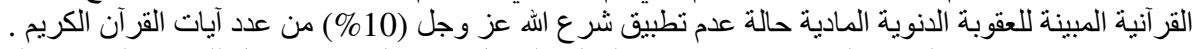
3.

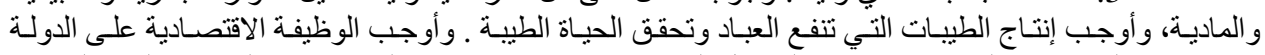

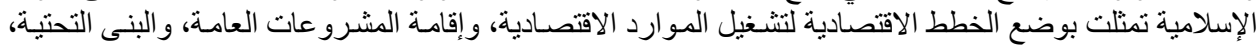

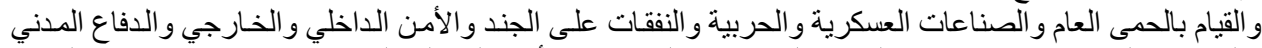

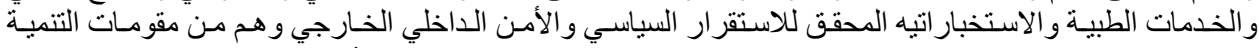

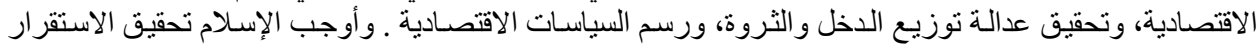

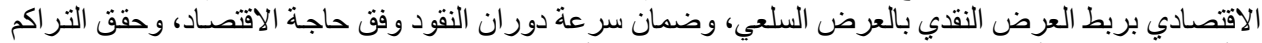

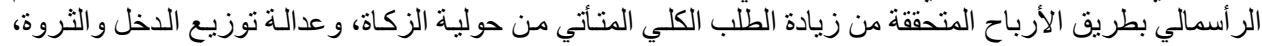

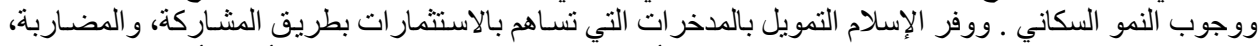

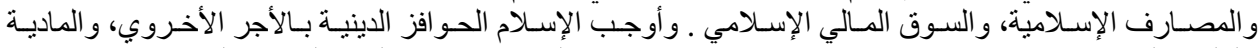

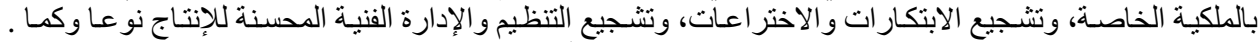

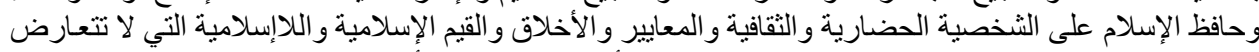

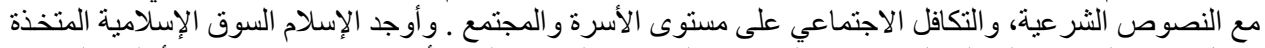

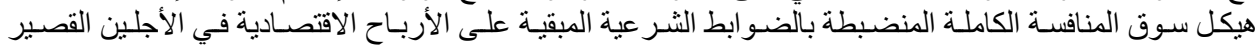

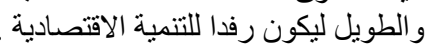

لاروطي الباحث تطبيق شر ع الله عز وجل لما فيه من تحقيق للسعادة التي تنع بها كافة المخلوقات الموجودة على الكرة

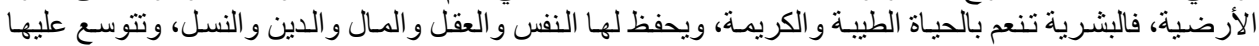

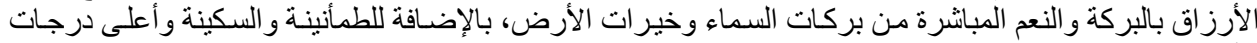

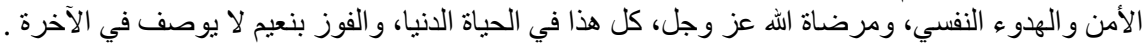

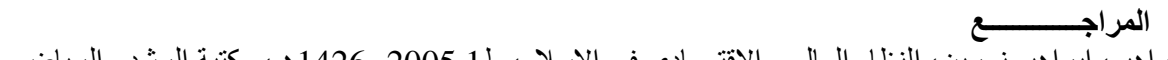

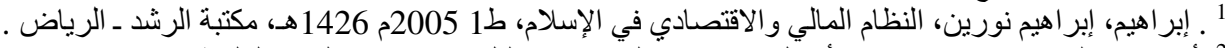

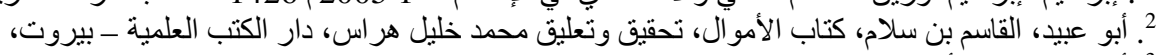

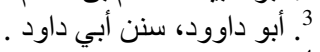

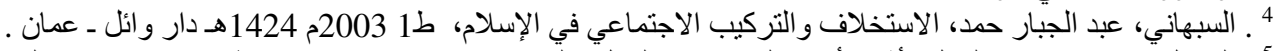

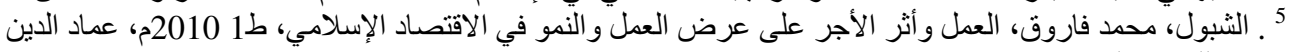

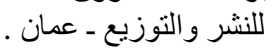
6. الثياني، محمد بن الحسن، الكسب ـ الاكتساب في الرزق المستطاب، ط 1 ـ 1980م، تحقيق وتقديم سهيل ركاز، نثر

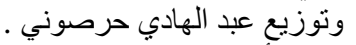

7. الثاطبي، أبو إبحق إبر اهيم بن موسى، الموادئ الفقات في أصول الثريعة، حقق و علق عليه : خالد عبد الفتاح شبل، مؤسسة

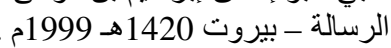
8. القرطبي، أبو عبد الله محمد بن أحمد الأنصاري، الجامع لأحكام القرآن، المكتبة التوفيقية ــ القاهرة .

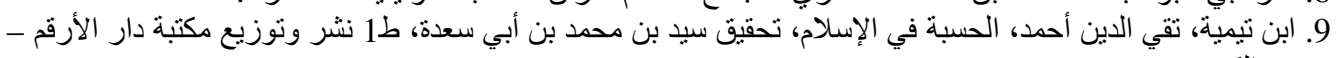

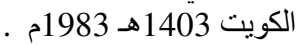

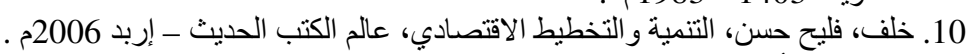

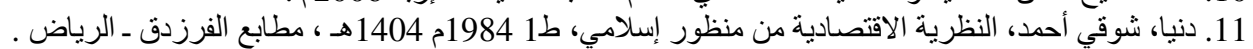

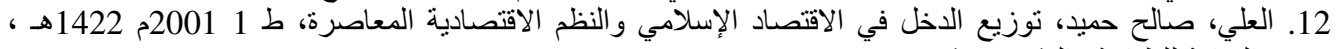

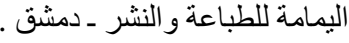

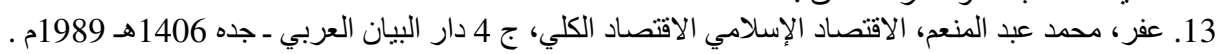

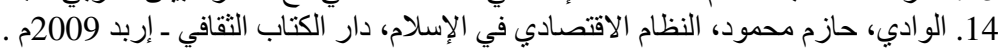

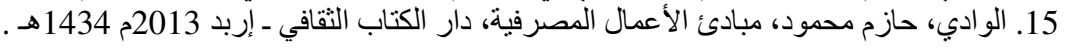


The theory of development in Islamic Economy

\section{References}

Ibrahim Noreen, The System of monetary and economic inIslamic, 1ed. 1424 - Alriad . Abu OpaidALqasim, Money Book, Berute .

Abu Daawood, Sunan Abu Daawood .

Affer Mohammad, Islamic Economy Macro economic, Jeddah 1989

Al-Ali Saleh, Allocation Income in Islamic and other economic system, 1ed. 2001, sera . Al-Kurtobi Mohammed, Aljameh for science Holly Quran, Egypt .

ALshatiby, Ibrahim, Al-muafaqat . Al-shebool Mohammed, Labor and effect wages on supply labor and economic growth on Islamic economy, 1ed. 2010 Amman .

Alshibanee Mohammed, Alkaseb, 1ed. 1980 Egypt .

Alwadihazem, The system of economy in Islamic, Jordan 2009.

Alwadihazem, Principle of Banking Operations, Jordan 2013.

Chaleffaleh, Development and planning economy, Jordan 2001 .

Doniashuqi, The Theory Economic in Islam, Al-raid 1984 .

IbenTaimiah, Alhessba In Islam, Alkuate 1983. 